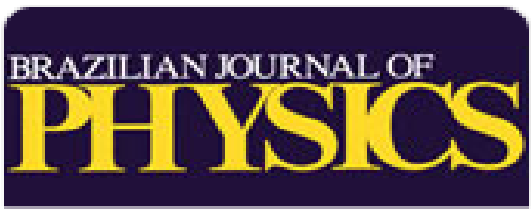

Brazilian Journal of Physics

ISSN: 0103-9733

luizno.bjp@gmail.com

Sociedade Brasileira de Física

Brasil

Cervantes, L. M.; Zúñiga, A.; Magaña, L. F.; Murillo, J. G.

Influence of a Time-Dependent Applied Electric Field on the Photocurrent in Photorefractive Materials

Brazilian Journal of Physics, vol. 44, núm. 4, 2014, pp. 334-339

Sociedade Brasileira de Física

Sâo Paulo, Brasil

Available in: http://www.redalyc.org/articulo.oa?id=46431147005

How to cite

Complete issue

More information about this article

Journal's homepage in redalyc.org

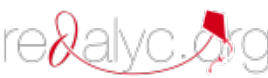

Scientific Information System

Network of Scientific Journals from Latin America, the Caribbean, Spain and Portugal

Non-profit academic project, developed under the open access initiative 


\title{
Influence of a Time-Dependent Applied Electric Field on the Photocurrent in Photorefractive Materials
}

\author{
L. M. Cervantes $\cdot$ A. Zúñiga $・$ L. F. Magaña $\cdot$ \\ J. G. Murillo
}

Received: 2 December 2011 Published online: 22 May 2014

(C) Sociedade Brasileira de Física 2014

\begin{abstract}
From the differential material rate equations, we found an additional term to the usual expression of the photocurrent, which appears only when a time-dependent external electric field is applied. This term influences the photocurrent in the material. We applied our equations to a $\mathrm{Bi}_{12} \mathrm{SiO}_{20}$ sample, under an applied sinusoidal electric field. This sample is illuminated with an oscillating interference pattern formed by two plane light waves, one of which is phase-modulated with frequency $\omega$. We found good agreement with experimental results. Besides, for this case, our prediction for the photocurrent is better than the usual prediction of the photocurrent for low values of $\omega$.
\end{abstract}

Keywords Photorefractive gratings $\cdot$ Photo-EMF . Non-linear-optics

L. M. Cervantes

Escuela Superior de Cómputo, Instituto Politécnico Nacional, Unidad Profesional Adolfo López Mateos, Código Postal 07730 Mexico, Distrito Federal, Mexico

\author{
A. Zúñiga $(\bowtie)$ \\ Escuela Superior de Física y Matemáticas, Instituto Politécnico \\ Nacional, Edificio 9, Unidad Profesional Adolfo López Mateos, \\ Código Postal 07738 Mexico, Distrito Federal, Mexico \\ e-mail: azuniga@esfm.ipn.mx

\section{F. Magaña} \\ Instituto de Física, Universidad Nacional Autónoma de México, \\ Apartado Postal 20-364, Código Postal 01000 Mexico, Distrito \\ Federal, Mexico \\ J. G. Murillo \\ Centro de Investigación en Materiales Avanzados, Miguel de \\ Cervantes120, Complejo Industrial Chihuahua, Código Postal \\ 31109 Chihuahua, Chihuahua, Mexico
}

\section{Introduction}

As it is well known, in photorefractive crystals, a light pattern may be transposed into a refractive index pattern. Inhomogeneous illumination excites charge carriers into conduction or valence band. The charge carriers migrate and spatially modulated currents arise, and the carriers are trapped in the dark regions of the crystal. Due to this charge distribution, a space charge field builds up and modulates the refractive index via the electrooptic effect. The amplitude of this space charge grating can be enhanced by using a nonstationary mechanism of recording. Two routes have been devised with this subject: by applying an external dc field to the crystal illuminated with a running interference pattern [1], and applying an ac electric field along the stationary interference pattern [2]. One of the most successful techniques is based on the so-called photoelectromotive force (photo-EMF). This method does not involve light diffraction from the recorded hologram and can be used for characterization of inorganic crystalline, and amorphous materials [3, 4]. The photo-EMF is the holographic alternating electric current in a semiconductor crystal illuminated by an oscillating interference pattern $[5,6]$. The most important feature of this technique is the interaction of the photoinduced space charge field grating with the grating of mobile photocarriers. This results in a periodic current flowing through the sample. This nonstationary photocurrent can be considered as the alternative optical technique for the study of the space charge grating formation in semiconductors. The presence of an external alternating electric field on the photoEMF effect is quite useful for characterization of hybrid materials $[7,8]$.

In this paper, we find from the differential material rate equations an additional term to the usual expression of the photocurrent. This term appears only when a time-dependent external electric field is applied and influences the photocurrent in the material. In order to assess the importance of this 
new term, we compare our predictions with experimental results. More specifically, we apply our equations to a $\mathrm{Bi}_{12} \mathrm{SiO}_{20}$ sample. This sample was under an applied sinusoidal electric field and illuminated with an oscillating interference pattern formed by two plane light waves, one of which is phase-modulated with frequency $\omega[6]$.

\section{Theoretical Framework}

We restrict our consideration to the band transport model considering electrons to be the only charge carriers and a single photoactive impurity level. The photorefractive response to illumination is described by the coupled nonlinear material rate equations $[9,10]$

$\frac{\partial N_{\mathrm{D}}^{+}}{\partial t}=s I\left(N_{\mathrm{D}}-N_{\mathrm{D}}^{+}\right)-\gamma n_{e} N_{\mathrm{D}}^{+}$

$\frac{\partial n_{e}}{\partial t}-\frac{\partial N_{\mathrm{D}}^{+}}{\partial t}=\frac{1}{e} \frac{\partial j}{\partial x}$

$j=e \mu n_{e} E+\mu k_{\mathrm{B}} T \frac{\partial n_{e}}{\partial x}$

$\frac{\partial E_{\mathrm{SC}}}{\partial x}=\frac{\partial E}{\partial x}=\frac{e}{\varepsilon_{0} \varepsilon}\left(N_{\mathrm{D}}^{+}-N_{\mathrm{A}}-n_{e}\right)$,

where $E=E_{\mathrm{SC}}(x, t)+E_{\mathrm{A}}(t), E_{\mathrm{SC}}$ is the space charge field, and $E_{\mathrm{A}}$ is the applied field; $s$ is the photoionization cross section, $\gamma$ the charge carrier recombination rate, $e$ the magnitude of electron charge, $\mu$ the mobility of the charge carrier, $N_{\mathrm{D}}$ the donor density, $N_{\mathrm{D}}^{+}$the ionized donor density, $N_{\mathrm{A}}$ the acceptor density, $n_{e}$ the charge carrier density, $j$ the electric current density, $k_{\mathrm{B}}$ the Boltzmann constant, $T$ the temperature, $t$ the time, $\varepsilon$ the dielectric constant, $\varepsilon_{0}$ the free space permittivity, and $I$ the light intensity.

In order to calculate the current density flowing through the sample, let us consider the continuity equation:

$\frac{\partial \rho(x, t)}{\partial t}=-\frac{\partial j(x, t)}{\partial x}$

Using Eq. (4) in Eq. (5) and integrating the resulting equation over the coordinate $x$, we obtain

$\frac{\partial}{\partial t}\left(\varepsilon \varepsilon_{0} E\right)=-j(x, t)+j_{0}(t)$.
We have introduced a time-dependent integration constant, which we call $j_{0}(t)$. Integrating Eq. (6) again over time yields

$\varepsilon \varepsilon_{0} E_{\mathrm{SC}}(x, t)=-\varepsilon \varepsilon_{0} \int_{0}^{t} \frac{d E_{\mathrm{A}}\left(t^{\prime}\right)}{d t^{\prime}} d t^{\prime}-\int_{0}^{t} j\left(x, t^{\prime}\right) d t^{\prime}+\int_{0}^{t} j_{0}\left(t^{\prime}\right) d t^{\prime}$.

We have considered that

$E_{\mathrm{SC}}(t)=0$, at $t=0$.

For the sinusoidal interference pattern (stationary, running, or oscillating), we have periodic boundary conditions. The averaging can be performed only over one spatial grating period $\Lambda$ [7], and with the help of Eq. (7), the boundary condition corresponding to the constraint of an applied voltage $V[11]$ :

$V=\int_{0}^{L} E(x, t) d x$

Assuming periodicity in the grating vector direction, we express the condition given in Eq. (8) over one grating period $\Lambda$ :

$V=N\left[\Lambda E_{\mathrm{A}}(t)\right]=\int_{0}^{N \Lambda} E(x, t) d x=N \int_{0}^{\Lambda} E(x, t) d x$

$\Lambda E_{\mathrm{A}}(t)=\int_{0}^{\Lambda} E(x, t) d x$, is:

$\frac{1}{\varepsilon \varepsilon_{0} \Lambda} \int_{0}^{\Lambda}\left[\int_{0}^{t} j_{0}\left(t^{\prime}\right) d t^{\prime}-\int_{0}^{t} j\left(x, t^{\prime}\right) d t^{\prime}-\varepsilon \varepsilon_{0} \int_{0}^{t} \frac{d E_{\mathrm{A}}\left(t^{\prime}\right)}{d t^{\prime}} d t^{\prime}\right] d x=0$.

Changing the order of integration, we have that

$\int_{0}^{t}\left[j_{0}\left(t^{\prime}\right)-\frac{1}{\Lambda} \int_{0}^{\Lambda} j\left(y, t^{\prime}\right) d y-\varepsilon \varepsilon_{0} \frac{d E_{\mathrm{A}}\left(t^{\prime}\right)}{d t^{\prime}}\right] d t^{\prime}=0$.

Notice that

$\int_{0}^{t} \frac{1}{\Lambda} \int_{0}^{\Lambda} \frac{d E_{\mathrm{A}}\left(t^{\prime}\right)}{d t^{\prime}} d x d t^{\prime}=\frac{\Lambda}{\Lambda} \int_{0}^{t} \frac{d E_{\mathrm{A}}\left(t^{\prime}\right)}{d t^{\prime}} d t^{\prime}=\int_{0}^{t} \frac{d E_{\mathrm{A}}\left(t^{\prime}\right)}{d t^{\prime}} d t^{\prime}$.

Finally,

$j_{0}(t)=\frac{1}{\Lambda} \int_{0}^{\Lambda} e \mu n_{e}(y, t) E(y, t) d y+\varepsilon \varepsilon_{0} \frac{d E_{\mathrm{A}}(t)}{d t}$.

We can see that the rate of change of the applied field significantly affects the photocurrent (Eq. (10)). The usual expression of the photocurrent [7] does not include the rate of change of the applied field. 
Our procedure to calculate the photocurrent is the following. First, we solve numerically the coupled, nonlinear material rate using Eqs. (1) to (4). From these solutions, we get the values for the full space charge field $E_{\mathrm{SC}}(x, t), N_{\mathrm{D}}{ }^{+}(x, t)$, and the mobile carrier density $n_{e}(x, t)$. In our numerical solution, we are imposing periodic boundary conditions along the coordinate $x$, with period $\Lambda$. We are not performing any Fourier expansion in our solution. We are obtaining, from our numerical procedure, the full overall solution for $E_{\mathrm{SC}}, N_{\mathrm{D}}{ }^{+}$, and $n_{e}$. This implies that we have in our solution all the Fourier components for these variables. The reader may see [11] for details of the numerical method. Then, we calculate the effective value (root mean square (RMS)) of $j_{0}(t)$, as given in Eq. (10).

The set of nonlinear partial differential Eqs. (1) to (4) constitutes a parabolic system. We solve this system numerically, with the constraint given by Eq. (8), and calculate the current from Eq. (10), from time equal to zero up to the stationary state. We followed the method of lines and used a finite element collocation procedure (with piecewise polynomials as the trial space) for the discretization of the spatial variable $x$. We used time-independent second-degree polynomials for this discretization. The collocation procedure reduces the system to a semidiscrete system (actually an initial value ordinary differential equation system), which then depends only on the time variable $t$. The time integration is then accomplished by use of slightly modified standard techniques. The approximate solution at any time is a second-order polynomial over each subinterval $\left(x_{n}, x_{n+1}\right)$. The coefficients of the terms depend only on time. We imposed the conditions of continuity of the polynomials and their derivatives on each extreme of the subinterval. The number of subintervals depends on the value of light modulation $m$. The larger the value of $m$, the larger the number of subintervals we had to take. For example, for $m=$ 0.01 , only 12 subintervals are required. For $m=0.9,45$ intervals are required.

The appropriate step sizes and time integration formulas were selected dynamically to maintain time discretization error below a specified level. For the initial part of the process, we used a time interval, to update the value of the current density and then the value of $J_{0}$, around the carrier lifetime/ 200 . The carrier lifetime is around $6 \times 10^{-6} \mathrm{~s}$. Then, we used a time interval around the carrier lifetime/ 2 up to the stationary state (around $4 \mathrm{~s}$ of total time).

On the other hand, for an oscillating interference pattern formed by two plane light waves of wavelength $\lambda$, one of which is being phase-modulated with frequency $\omega$ and amplitude $\Delta$, the light intensity is

$I(x, t)=I_{0}\left[1+m \cos \left(K_{G} x+\Delta \cos (\omega t)\right)\right]$

where $I_{0}$ is the average light intensity, $m$ is the modulation depth, $\vec{K}_{G}=2 \pi / \Lambda$ is the grating vector of the interference pattern, and $\Lambda$ is the fringe spacing.
The above-described numerical method does not require to have small values of the contrast $m$ and of the amplitude of phase modulation $\Delta$.

Now, let us write Eq. (10) as

$j_{0}(t)=j_{S}(t)-\sqrt{2} \Omega E_{0} \varepsilon \varepsilon_{0} \sin (\Omega t)$

where $j_{s}(t)$ is the usual current density term [7] and $\Omega$ is the frequency of oscillation of the applied electric field. Considering an oscillating interference period $T_{\omega}=2 \pi / \omega$, clearly, the effective density current approximately depends on the frequency as

$j_{\mathrm{RMS}}^{2} \approx \frac{1}{T_{\omega}} \int_{0}^{T_{\omega}} j_{S}^{2} d t-2 \sqrt{2} j_{S} \Omega^{2} E_{0} \varepsilon \varepsilon_{0} \pi\left(\frac{1}{\omega}\right)$,

where we find relied on the approximations $(1+x)^{2} \approx 1+2 x$ and $T_{\omega}$

$\frac{1}{T_{\omega}} \int_{0}^{T_{\omega}} \sin (\Omega t) d t \approx \frac{\pi \Omega}{\omega}$.

Notice that if the modulation frequency $\omega$ increases in Eq. (12), the additional term decreases. Therefore, for large frequencies, the correction to the usual expression of the photocurrent goes to zero.

\section{Results and Discussion}

In order to assess the importance of the additional term on the photocurrent, we compare our predictions with experimental results given in [6]. In this reference, we have measurements for the photorefractive $n$-type $\mathrm{Bi}_{12} \mathrm{SiO}_{20}$ crystal (BSO) illuminated with an oscillating interference pattern given by Eq. (11). The sample is under the action of an applied alternating electric field. Figure 1 shows a diagram of the experimental setup used to make these measurements, as was reported in reference [6]. The parameters for BSO are from [6, 12] and are given in Table 1.

Additional required information to apply our equations to the experiment from [6] is the following: The crystal dimensions $1 \times 3 \times 10 \mathrm{~mm}^{3}$. The interelectrode spacing was $1 \mathrm{~mm}$. Two silver paste electrodes were painted on lateral surfaces of $3 \times 4 \mathrm{~mm}^{2}$. The front surface of the crystal $\left(1 \times 10 \mathrm{~mm}^{2}\right)$ was polished and illuminated by two laser beams (with a wavelength $\lambda=442 \mathrm{~nm}$ ), one of which is phase-modulated with frequency $3 \leq \omega / 2 \pi \leq 2,000(\mathrm{~Hz})$ and amplitude $\Delta=0.16$. The resulting oscillating interference pattern exhibited a power $P_{0}=0.19 \mathrm{~mW}$ with contrast $m=0.65$. In fact, the light intensity was $I_{0}=P_{0} / A \approx 5 \mathrm{~mW} / \mathrm{cm}^{2}$, since the light pattern and electric field were simultaneously present only on the surface 


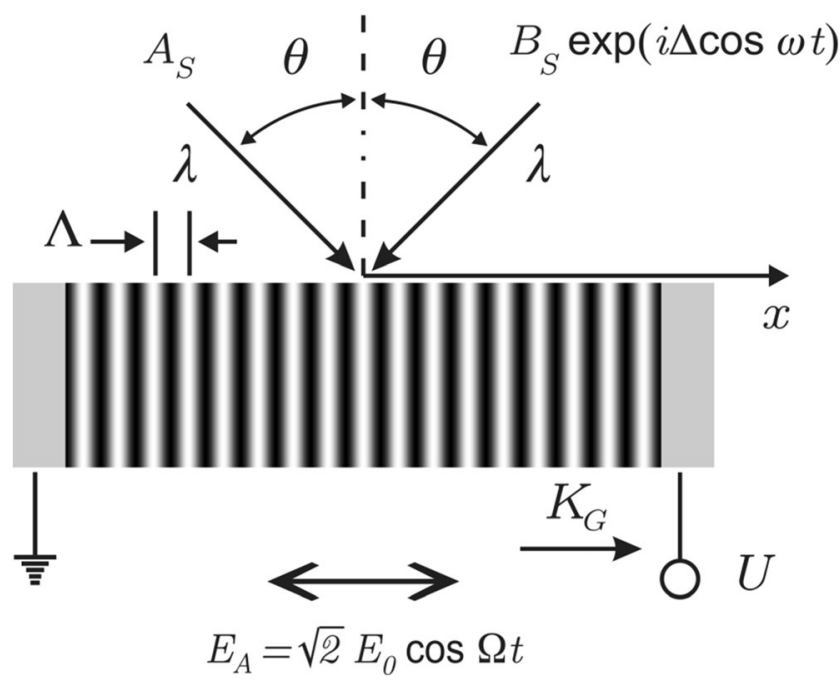

Fig. 1 Typical experimental setup used for the photo-EMF measurements. The waves $A_{S}$ and $B_{S} \exp [i \Delta \cos (\omega t)]$ interfere inside the BSO crystal. A conventional transmission configuration is employed. The grating vector $\vec{K}_{G}$ is perpendicular to the electrode's planes

area $A=4 \mathrm{~mm}^{2}$. The angle of incidence $\theta$ is given by the Bragg relation $\Lambda=\lambda / 2 \sin \theta$. The fringe spacing is $\Lambda \approx 125 \mu \mathrm{m}$ $\left(\vec{K}_{G}=5 \times 10^{4} \mathrm{~m}^{-1}\right)$. The applied ac field is parallel to the vector $\vec{K}_{G}$, with frequency $\Omega / 2 \pi=6.0 \mathrm{kHz}$.

Finally, it is worth mentioning that the experiment considered the case of the "relaxation-type" semiconductor, i.e., when the relation between the Maxwell relaxation time $\tau_{\mathrm{M}}$ and lifetime of photocarriers $\tau<<\tau_{\mathrm{M}}$, should be satisfied. To fulfill this condition, additional infrared illumination of the crystal was used $\left(\lambda_{\mathrm{IR}}=650-2,700 \mathrm{~nm}, I_{\mathrm{IR}}=13 \mathrm{~mW} / \mathrm{mm}^{2}\right)$. The parameters $\tau \approx 12 \mu$ s and $\tau_{\mathrm{M}} \approx 0.25 \mathrm{~ms}$ used in our numerical method were obtained from an experimental measurement of nonstationary photocurrent with additional infrared illumination [6]. In our numerical solution, the initial carrier density $n_{e}(x, t=0)=\varepsilon \varepsilon_{0} / e \mu \tau_{\mathrm{M}}$ is generated by the infrared illumination.

In Fig. 2, we compare of our results with the calculations using Eq. (18) of [6], and with experimental data from [6] for the photo-EMF, as a function of the frequency $\omega$. The experimental data are for applied fields of $0(\circ), 0.53(\bullet), 1.3(\square)$,

Table 1 Experimental parameters $[6,12]$ for BSO for our calculations

\begin{tabular}{lll}
\hline & BSO & \\
\hline$\varepsilon$ & Dielectric constant & 56 \\
$\Lambda$ & Fringe spacing $(\mu \mathrm{m})$ & 125 \\
$\mu \tau$ & Mobility lifetime product $\left(\mathrm{m}^{2} \mathrm{~V}^{-1}\right)$ & $1.2 \times 10^{-10}$ \\
$\gamma$ & Recombination constant $\left(\mathrm{m}^{3} \mathrm{~s}^{-1}\right)$ & $1.6 \times 10^{-17}$ \\
$s$ & Photoionization cross section $\left(\mathrm{m}^{2} \mathrm{~J}^{-1}\right)$ & $1.0 \times 10^{-5}$ \\
$N_{\mathrm{D}}$ & Donor density $\left(\mathrm{m}^{-3}\right)$ & $10^{25}$ \\
$N_{\mathrm{A}}$ & Acceptor density $\left(\mathrm{m}^{-3}\right)$ & $10^{22}$ \\
$\Delta$ & Beam-modulated amplitude & 0.16 \\
\hline
\end{tabular}

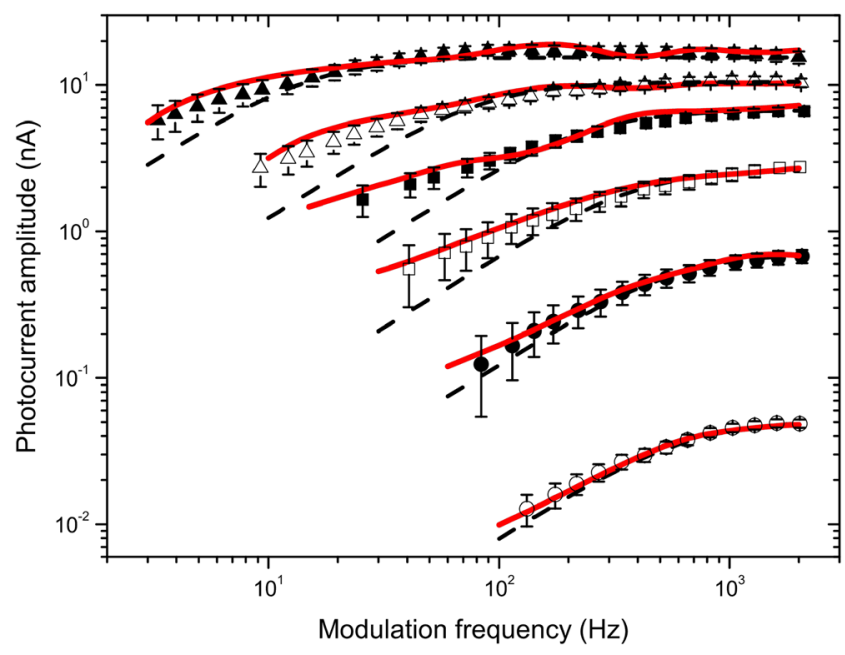

Fig. 2 Amplitude of the photo-EMF as a function of the oscillation frequency of the interference grating for different amplitudes of applied electric fields. White circle indicates $0 \mathrm{kV} / \mathrm{cm}$, black circle $0.53 \mathrm{kV} / \mathrm{cm}$, white square $1.3 \mathrm{kV} / \mathrm{cm}$, black square $2.1 \mathrm{kV} / \mathrm{cm}$, white triangle $4.1 \mathrm{kV} /$ $\mathrm{cm}$, and black triangle $10 \mathrm{kV} / \mathrm{cm}$. The solid lines are our calculations in accordance with the effective value (RMS) of our Eq. (12). Dashed lines are results for the photocurrent amplitude $\left|J^{\omega}\right|$ directly calculated from Eq. (18) of reference [6]

$2.1(\bullet), 4.1(\Delta)$, and $10 \mathrm{kV} / \mathrm{cm}(\mathbf{\Delta})$. Our results, in accordance with the effective value (RMS) of Eq. (10), are the solid lines. The calculations of the photocurrent amplitude $\left|J^{\omega}\right|$ from Eq. (18) of [6] are the dashed lines. Notice that our results are in very good agreement with experimental results for all values of frequencies and applied fields. Besides, we can see a concurrence between our calculations, the results from Eq. (18) in [6], and experimental data only for the region of large frequencies (above $10^{2} \mathrm{~Hz}$ ). For lower values of

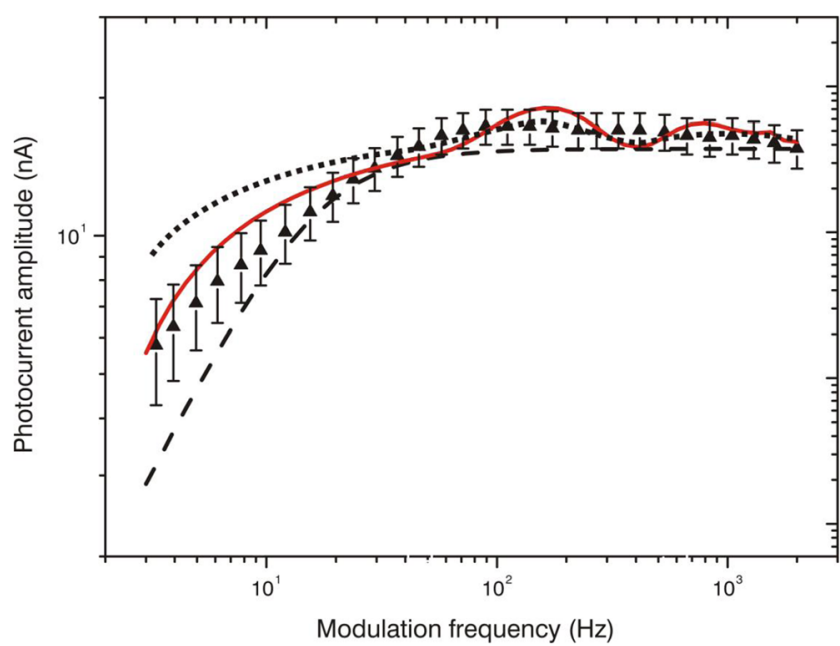

Fig. 3 The amplitude of the photo-EMF as a function of the oscillation frequency of the interference grating for an applied electric field of $10 \mathrm{kV} /$ $\mathrm{cm}$. Black triangle represents the experimental results. The solid line refers to our calculations in accordance with the effective value (RMS) of our Eq. (12). The dotted line corresponds to our Eq. (12) but without the displacement current contribution. Dashed lines are results for the photocurrent amplitude $\left|J^{\omega}\right|$ directly calculated from Eq. (18) of reference [6] 
Fig. 4 The photocurrent amplitude versus scaled time for different amplitudes of applied alternating fields $E_{0}$ and oscillation frequency $\omega / 2 \pi=2,000 \mathrm{~Hz} ; T_{\omega}=2 \pi / \omega$ (a) $\mathrm{E}_{0}=0 \mathrm{kV} / \mathrm{cm}$

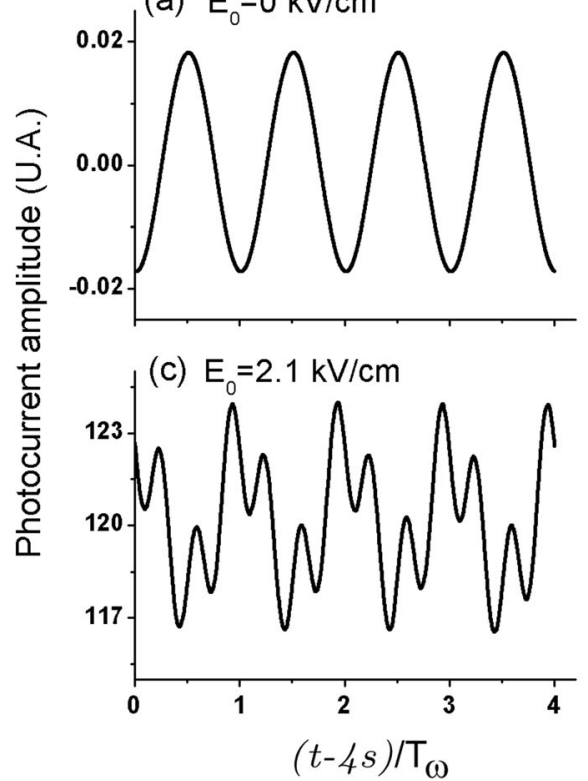

(b) $E_{0}=1.3 \mathrm{kV} / \mathrm{cm}$

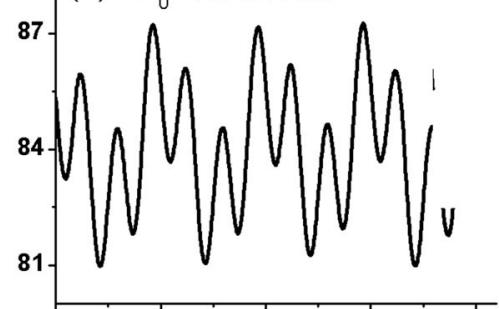

(d) $E_{0}=10 \mathrm{kV} / \mathrm{cm}$

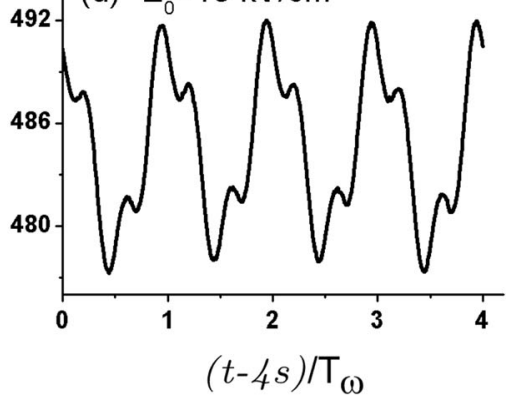

frequency, our predictions are closer to the experimental measurements than the values coming from Eq. (18) of [6]. This occurs for all the values of the applied electric field. This can be easily understood using Eq. (12) given in "Section 2". Clearly, the second term in Eq. (12) improves the prediction of the photocurrent amplitude for low frequencies and different values of applied fields.

Furthermore, notice that for large applied fields our numerical results have small oscillations around the experimental data. We expect these oscillations to be observed. They are larger than the experimental error bars. Notice that our solution is for the full space charge field. Our method does not rely on Fourier's expansion.

In Fig. 3, we make a comparison of the experimental results (A) of the amplitude of the photo-EMF as a function of the oscillation frequency of the interference grating for an applied electric field of $10 \mathrm{kV} / \mathrm{cm}$ with our calculation of $J_{0}$, without the displacement current term (dotted line), and with $J_{0}$ given by our Eq. (12) (solid line). Clearly, the inclusion of the displacement current is necessary to explain the experimental results. Dashed lines are results for the photocurrent amplitude $\left|J^{\omega}\right|$ directly calculated from Eq. (18) of reference [6].

Finally, in Fig. 4, we exhibit for the given experimental conditions the particularly strong contribution of the Fourier component of the applied field with frequency $\Omega=3 \omega$ to the photo-EMF. In this figure, we present our results for the total photocurrent amplitude versus time for an oscillation frequency $\omega / 2 \pi=2,000 \mathrm{~Hz}$ and different applied alternating field amplitudes $E_{0}$ : (a) 0 , (b) 1.3 , (c) 2.1 , and (d) $10 \mathrm{kV} / \mathrm{cm}$, respectively. Notice that the scale in the vertical axis is different in (a), (b), (c), and (d). For example, the photocurrent in (a) has the maximum value of 0.02 and in (d) of 492. Contrary to the diffusion case, as the applied field amplitude is increased, we have an oscillating space charge field that strongly interacts with the carrier density. Hence, it gives a remarkable contribution to photo-EMF, as shown in (b)-(d).

\section{Conclusion}

We have found an additional term to the usual expression of the photocurrent, which appears only when an ac field is applied. We found that this term improves the prediction of the photocurrent. Our calculations are in very good agreement with experimental results. We applied our analysis to a BSO crystal illuminated with an oscillating interference pattern formed by two plane light waves, one of which is phase-modulated with frequency $\omega$. Besides, for this case, our prediction for the photocurrent is better than the usual prediction of the photocurrent for low values of $\omega$. The theory developed in this paper could be applicable to other photorefractive materials.

Acknowledgments LF Magaña thanks Dirección General de Asuntos del Personal Académico de la Universidad Nacional Autónoma de México partial financial support by Grant IN100111. A Zúñiga would like to thank useful discussions with A.V. Khomenko and N. Korneev, and SIPIPN grant 20140888.

\section{References}

1. P. Refregier, L. Solymar, H. Rajbenbach, J.P. Huignard, Two-beam coupling in photorefractive $\mathrm{Bi}_{12} \mathrm{SiO}_{20}$ crystals with moving grating: theory and experiments. J. Appl. Phys. 58, 45-57 (1985)

2. S.I. Stepanov, M.P. Petrov, Efficient unstationary holographic recording in photorefractive crystals under an external alternating electric field. Opt. Commun. 53, 292-295 (1985) 
3. M.P. Petrov, S.I. Stepanov, A.V. Khomenko, Photorefractive crystals in coherent optical systems (Springer, Berlin, 1991)

4. S.I. Stepanov, V.V. Kulikov, M.P. Petrov, Running holograms in photorefractive $\mathrm{Bi}_{12} \mathrm{SiO}_{20}$ crystals. Opt. Commun. 44, 19-23 (1982)

5. M.P. Petrov, I.A. Sokolov, S.I. Stepanov, G.S. Trofimov, Non-steady photo-electromotive-force induced by dynamic gratings in partially compensated photoconductors. J. Appl. Phys. 68, 2216-2225 (1990)

6. M. Bryushinin, V. Kulikov, I. Sokolov, Nonresonant enhancement of the nonstationary holographic currents in photoconductive crystals. Phys. Rev. B 65, 245204 (2002)

7. S. Stepanov, Photo-electromotive-force effect in semiconductors, in Handbook on advanced electronics and photonics materials, ed. by H.S. Nalwa (Academic, San Diego, 2001), pp. 205-272

8. S. Mansurova, K. Meerholz, E. Sliwinska, U. Hartwig, K. Buse, Enhancement of charge carrier transport by doping PVK-based photoconductive polymers with $\mathrm{LiNbO}_{3}$ nanocrystals. Phys. Rev. B 79, 174208 (2009)

9. N. Kukhtarev, T. Kukhtareva, P.P. Banerjee, A unified treatment of radiation-induced photorefractive, thermal, and neutron transmutation gratings. Proc. IEEE 87, 1857-1869 (1999)

10. N. Kukhtarev, V. Markov, S. Odulov, M. Soskin, V. Vinetskii, Holographic storage in electro-optic crystals. II. Beam couplinglight amplification. Ferroelectrics 23, 949-964 (1979)

11. J.G. Murillo, L.F. Magaña, M. Carrascosa, F. Agulló-López, Temporal evolution of the physical response during photorefractive grating formation and erasure for BSO. J. Appl. Phys. 78, 5686-5690 (1995)

12. L.F. Magaña, I. Casar, J.G. Murillo, Beam energy exchange in sillenite crystals $\left(\mathrm{Bi}_{12} \mathrm{SiO}_{20}\right.$ and $\left.\mathrm{Bi}_{12} \mathrm{TiO}_{20}\right)$, considering the variation of light modulation along sample thickness in a strong nonlinear regime. Opt. Mater. 30, 979-986 (2008) 